\title{
Tissue factor pathway inhibitor-2 is specifically expressed in ovarian clear cell carcinoma tissues in the nucleus, cytoplasm and extracellular matrix
}

\author{
YUKIHIDE OTA $^{1,2}$, SHIRO KOIZUME ${ }^{1}$, YOSHIYASU NAKAMURA ${ }^{1}$, MITSUYO YOSHIHARA $^{1}$, \\ TOMOKO TAKAHASHI ${ }^{1}$, SHINYA SATO ${ }^{1}$, SHOHEI MYOBA ${ }^{3}$, NORIHISA OHTAKE ${ }^{3}$, \\ HISAMORI KATO ${ }^{4}$, TOMOYUKI YOKOSE ${ }^{5}$, ETSUKO MIYAGI ${ }^{2}$ and YOHEI MIYAGI ${ }^{1}$
}

\author{
${ }^{1}$ Molecular Pathology and Genetics Division, Kanagawa Cancer Center Research Institute, Yokohama 241-8515; \\ ${ }^{2}$ Department of Obstetrics and Gynecology, Yokohama City University Graduate School of Medicine, Yokohama 236-0004; \\ ${ }^{3}$ Bioscience Division, Reagent Development Department, Tosoh Corporation, Ayase, Kanagawa 252-1123; \\ Departments of ${ }^{4}$ Gynecologic Oncology and ${ }^{5}$ Pathology, Kanagawa Cancer Center, Yokohama 241-8515, Japan
}

Received September 30, 2020; Accepted December 22, 2020

DOI: $10.3892 /$ or.2021.7944

\begin{abstract}
Tissue factor pathway inhibitor-2 (TFPI-2) is a promising candidate as a serum biomarker of ovarian clear cell carcinoma (OCCC), a lethal histological subtype of epithelial ovarian cancer (EOC). TFPI-2 is a secreted serine protease inhibitor that suppresses cancer progression through the inhibition of matrix protease activities. Previous studies have also identified TFPI-2 in the nucleus, and a possible function of nuclear TFPI-2 as a transcriptional repressor of matrix metalloproteinase-2 (MMP-2) was recently demonstrated. We are currently establishing TFPI-2 as a serum biomarker for OCCC patients; however, TFPI-2 expression in OCCC tissues has not been previously investigated. In the present study, we examined TFPI- 2 expression and its localization in 11 OCCC cell lines by western blotting and enzyme-linked immune assay. Four cell lines expressed TFPI-2 in the nucleus, cytoplasm and culture plate-attached extracellular fraction, while four other cell lines expressed TFPI-2 only in the extracellular fraction. In the remaining three cell lines, TFPI- 2 was not identified in any fraction. The amount of secreted soluble TFPI- 2 showed
\end{abstract}

Correspondence to: Dr Yohei Miyagi, Molecular Pathology and Genetics Division, Kanagawa Cancer Center Research Institute, Yokohama, 2-3-2 Nakao, Asahi-ku, Yokohama 241-8515, Japan E-mail: miyagi@gancen.asahi.yokohama.jp

Abbreviations: TFPI-2, tissue factor pathway inhibitor-2; OCCC, ovarian clear cell carcinoma; EOC, epithelial ovarian cancer; CA125, cancer antigen 125; PBS, phosphate-buffered saline; WCF, whole cell fraction; ECF, extracellular fraction; ECM, extracellular matrix; $\mathrm{CM}$, conditioned medium; AIA, automated immunoassay analyzer; $\mathrm{KCCH}$, Kanagawa Cancer Center Hospital; FFPE, formalin-fixed and paraffin-embedded; IHC, immunohistochemistry

Key words: TFPI-2, ovarian clear cell carcinoma, immunohistochemistry, biomarker, subcellular localization similar trends to that of the plate-attached fraction. We next investigated the expression levels and distribution of TFPI-2 in surgically resected EOC tissues by immunohistochemistry. In 52 of the $77(67.5 \%)$ OCCC tumors, TFPI- 2 expression was detected in at least one of the nuclear, cytoplasmic and extracellular matrix fractions. In contrast, we did not identify TFPI- 2 in the other EOC subtypes $(n=65)$. TFPI-2-positive expression distinguished CCC from the other EOC tissues with a sensitivity of $67.5 \%$ and specificity of $100 \%$. Although the inherent tumor suppressor function, statistical analyses failed to demonstrate correlations between TFPI-2 expression and clinical parameters, including 5-year overall survival, except for the patient age. In conclusion, we identified TFPI-2 expression in the nucleus, cytoplasm and extracellular matrix in OCCC tissues. The high specificity of TFPI-2 may support its use for diagnosis of OCCC in combination with existing markers.

\section{Introduction}

Ovarian cancer is the most lethal gynecological malignancy in developed countries (1). In 2018, approximately 295,400 new cases of ovarian cancer were diagnosed and 184,800 patients with ovarian cancer died worldwide (2). Clear cell carcinoma (CCC) is one of the common histological types of epithelial ovarian cancer (EOC) (3). The frequency of ovarian CCC (OCCC) varies depending on ethnicity; CCC accounts for 11.7-26.9\% of Japanese EOC cases in comparison with 4.6-8.4\% of EOC in North America $(4,5)$. About half of the OCCC cases are diagnosed at stage I and have a good prognosis (6). However, advanced stage or recurrent OCCC cases have worse prognosis than the other EOC subtypes due to the resistance to standard platinum-based chemotherapy (7). Therefore, early detection and complete resection are crucial in OCCC treatment. Cancer antigen 125 (CA125) is currently the most frequently used serum biomarker for EOC. However, CA125 is also elevated in benign conditions such as endometrial cyst and peritonitis, menstruation and other intra-abdominal 
malignancies (8). Thus, CA125 does not necessarily distinguish malignancy. Additionally, CA125 often fails to detect OCCC even at advanced stages (9).

Tissue factor pathway inhibitor-2 (TFPI-2) protein, a homologue of tissue factor pathway inhibitor (TFPI), is a secreted protease inhibitor containing an $\mathrm{N}$-terminal signal peptide and Kunitz-type serine protease inhibitory domains (10). Despite its structural similarity to TFPI, TFPI-2 has weak inhibitory activity against the tissue factor blood coagulation pathway, which is initiated by the serine protease tissue factor-coagulation factor VIIa complex, and instead inhibits a wide variety of serine proteases, such as plasmin, plasma kallikrein, trypsin and chymotrypsin (10). TFPI-2 is predominantly and highly expressed in placenta $(11,12)$. Although several studies have examined the association between TFPI- 2 and preeclampsia $(13,14)$, the biological function of TFPI-2 is not fully understood.

Many reports have shown that TFPI-2 is genetically silenced in aggressive cancers, such as glioma (15), non-small cell lung cancer (16), pancreatic cancer (17), breast cancer (18), malignant melanoma (19) and hepatocellular carcinoma (20), indicating its tumor-suppressor character. The anticancer functions of TFPI-2 are generally thought to be mediated by its protease inhibitory activities, which lead to inhibition of cell proliferation, invasion or angiogenesis and augmentation of apoptosis $(21,22)$. Recent studies also suggest another tumor-suppressor aspect of TFPI-2, demonstrating that exogenously applied TFPI- 2 localized in the nucleus of fibrosarcoma cells (23) and overexpressed TFPI-2 in breast cancer cells negatively regulate matrix metalloproteinase- 2 (MMP-2) expression (24).

In contrast to the results showing epigenetic silencing of TFPI-2 in several tumor types, we recently reported that cultivated OCCC cells produce and secrete TFPI-2 into medium and we initiated studies to develop TFPI-2 as a specific serum biomarker for preoperative clinical diagnosis for OCCC $(25,26)$. Serum TFPI-2 level discriminated CCC from other histological types of EOC and endometrial cyst (26), which is a risk factor for CCC (27). Although we are considering that serum TFPI-2 is derived from OCCC tumor cells, TFPI-2 expression was also reported in endothelial cells, which are distributed throughout the body (23). Furthermore, non-secreted fractions of TFPI-2 were reported in in vitro studies in other tumor types. Therefore, in the present study, we examined TFPI-2 expression and localization of TFPI-2 in multiple OCCC cell lines and in surgically removed OCCC tissues including tissues of other EOC histologic types. We also investigated the association between TFPI-2 expression and clinical characteristics of OCCC patients to clarify the role of TFPI-2 in OCCC.

\section{Materials and methods}

Cell lines and cell culture. The OCCC cell lines ES-2 (ATCC CRL-1978) and TOV-21G (ATCC CRL-11730) were purchased from the American Type Culture Collection. OVISE (JCRB1043), OVMANA (JCRB1045), OVTOKO (JCRB1048), RMG-1 (JCRB0172) and HAC-2 (JCRB1359) cells were obtained from JCRB Cell Bank. JHOC-5 (RCB1520), JHOC-7 (RCB1688), JHOC-8 (RCB1723) and
JHOC-9 (RCB2226) cell lines were from RIKEN Bioresource Center Cell Bank. These OCCC cell lines were maintained in RPMI-1640 medium supplemented with $10 \%$ fetal bovine serum (FBS) and penicillin-streptomycin at $37^{\circ} \mathrm{C}$ in a humidified atmosphere of $5 \% \mathrm{CO}_{2}$.

Preparation of subcellular fractions. Cells were cultured for 2 days in $100-\mathrm{mm}$ plates until they reached semi-confluency. Cells were washed with phosphate-buffered saline (PBS) and then dissociated using Accutase reagent (Nacalai Tesque) according to the manufacturer's instruction. Dissociated cells were collected to prepare the whole cell fraction (WCF). Plates were rinsed twice with PBS, and the fraction that remained attached to the plate was collected by scraping the plates with lysis buffer and was considered the extracellular fraction (ECF). (NuPAGE NP0007, Thermo Fisher Scientific, Inc.). The Nuclear Extract Kit (Active Motif Inc.) was used for preparation of cytoplasmic and nuclear fractions from WCFs according to the manufacturer's instructions. Cells were cultured with $10 \mathrm{ml}$ of RPMI-1640 medium supplemented with $10 \%$ FBS and penicillin-streptomycin for 2 days in $100-\mathrm{mm}$ plates. Culture medium of semi-confluent cells was collected and centrifuged at $180 \mathrm{x}$ g for $3 \mathrm{~min}$. The supernatant was obtained as conditioned medium (CM).

Western blotting. Western blotting was performed using the NuPAGE $4-12 \%$ gradient Bis-Tris Protein Gel system (Thermo Fisher Scientific, Inc.) with MOPS running buffer (Thermo Fisher Scientific, Inc.). To detect TFPI-2, we used mouse monoclonal anti-TFPI-2 antibody (clone 28Aa, $1 \mu \mathrm{g} / \mathrm{ml}$, diluted 1:2,000) raised against a synthetic peptide antigen corresponding to the N-terminal of mature TFPI-2 protein after cleavage of the putative signal peptide (13). Anti-vinculin (V9131, diluted 1:10,000, Sigma-Aldrich; Merck KGaA), anti-Lamin A (sc-20680, diluted 1:500, Santa Cruz Biotechnology, Inc.) and anti- $\alpha$-tubulin antibodies (T-9026, diluted 1:3,000, Sigma-Aldrich; Merck KGaA) were used for protein loading controls. Secondary antibody reaction was performed with peroxidase-conjugated anti-mouse IgG (NA931, 1:100,000, Cytiva) or anti-rabbit IgG (NA934, 1:100,000, Cytiva). Detection was performed using the ImmunoStar LD enhanced chemiluminescence detection reagent (FUJIFILM Wako Chemicals).

TFPI- 2 concentration in CM. The TFPI- 2 concentration in $\mathrm{CM}$ was measured on an automated immunoassay analyzer (AIA) system (TOSOH, Japan) as described previously (26). Briefly, measurement of TFPI-2 using the AIA system was completed as a sandwich-type, one-step immune fluorometric assay using two different anti-TFPI-2 monoclonal antibodies, one of which was coated on magnetic beads and the other was labeled with alkaline phosphatase. As the calibration standard of the assay, recombinant TFPI-2 protein was prepared from the CM of SP2/0 cells transfected with the TFPI-2 expression vector and spiked into sample dilution buffer.

Patients and sample collection. A total of 142 patients with a confirmed histopathological diagnosis of EOC at Kanagawa Cancer Center Hospital (KCCH), Japan were included in this study. Patients who underwent treatment before 
primary debulking surgery or exploratory laparotomy were excluded. Patients with other cancers were also excluded. We examined all 71 EOC patients who matched the criteria from 2014 to 2017 to evaluate the expression of TFPI-2 along with the histological subtypes. Due to the small number of the included cases, 8 patients with endometrioid carcinoma and 14 patients with mucinous carcinoma were selected from the period before 2014 and additionally examined. Formalin-fixed and paraffin-embedded (FFPE) tissue sectioned to $4 \mu \mathrm{m}$-thickness were prepared from archives of the Department of Pathology, KCCH. Whole tissue sections of tumors of all enrolled patients were analyzed. Representative non-neoplastic regions of the surgical specimens of EOC cases were also examined in 18 cases, including endometrium and fallopian tubal epithelium (CCC: 9, serous: 3, endometrioid: 3, mucinous: 3). Written informed consent for research using specimens derived from routine clinical procedures was obtained from all patients. The experimental protocol of the present study was reviewed and approved by the Institutional Review Board of KCCH (approval no. Ethics-2018-10).

Immunohistochemical analysis of TFPI-2 expression. FFPE tissue specimens on glass slides were routinely stained with hematoxylin and eosin. Deparaffinized and rehydrated slides were immersed in 0.01 M citrate, pH6.0 (Sigma-Aldrich; Merck $\mathrm{KGaA}$ ), and heat-induced antigen retrieval was performed in an autoclave at $110^{\circ} \mathrm{C}$ for $15 \mathrm{~min}$. Slides were cooled to room temperature, washed in PBS and immersed in $3 \% \mathrm{H}_{2} \mathrm{O}_{2}$ diluted in methanol. For primary antibody, 28Aa antibody was diluted to $5 \mu \mathrm{g} / \mathrm{ml}$. Histofine Simplestain Max PO (M) (Nichirei) and Histofine DAB Substrate kit (Nichirei) were used to detect the labeled antigens. Placental tissue was used as positive control for TFPI-2 staining (13). Non-specific mouse IgG was used as a negative control. We conducted an absorption test to evaluate the specificity of the staining. Antibodies were incubated with a 20-fold excess molar concentration of the antigen for $24 \mathrm{~h}$ prior to the primary antibody reaction (28). The antigen for the 28Aa antibody is the 14 amino acid residues corresponding to the N-terminus of mature TFPI-2 protein, NH2-DAAQEPTGNNAEIC-COOH (13), linked to keyhole limpet hemocyanin. We used another anti-TFPI-2 antibody B-7 (sc-48380, diluted 1:200, Santa Cruz Biotechnology, Inc.) for detection of nuclear TFPI-2. The B-7 antibody is a mouse monoclonal antibody that was raised against peptides corresponding to amino acid residues 71-190 of human TFPI-2. We also conducted an absorption test using placental tissue with recombinant full-length TFPI-2 protein (OriGene) as antigen. TFPI-2 protein staining (cytoplasmic and nuclear staining) was scored by the H-score method (29). Briefly, the $\mathrm{H}$-score was calculated as the sum of the products of multiplying the staining intensity $(0,1+, 2+, 3+)$ by percentage stained area. For example, in a case with the intensity and percentage staining of $0+: 70 \%, 1+: 20 \%, 2+: 10 \%$ and $3+: 0 \%$, the $\mathrm{H}$-score is calculated as $40(40=0 \times 70+1 \times 20+10 \times 2+0 \times 3)$. Under a pathologist supervision, automated scoring on tumor regions was performed using Aperio's annotation software 'Aperio Cytoplasm Algorithm' (Leica Biosystem). We defined the cut-off value for TFPI-2 positivity as an H-score of 1 to reduce false negatives. We evaluated TFPI-2 expression within extracellular matrix (ECM) as 'positive' or 'negative.' We analyzed TFPI-2 expression and clinical characteristics of the OCCC patients.

Statistical analysis. Statistical analysis was performed using IBM SPSS Statistics 19 software (IBM Corp.). Clinicopathological parameters were evaluated using Kruskal-Wallis test or Mann-Whitney U test for continuous variables and Fisher's exact test for non-continuous variables. Relationships between TFPI-2 expression and 5-year overall survival were estimated by Kaplan-Meier method and compared by log rank test. Cox regression analysis was used for multivariate analysis of 5-year overall survival. $\mathrm{P}<0.05$ was considered to indicate a statistically significant difference.

\section{Results}

Expression, subcellular localization and secretion of TFPI-2 in OCCC cell lines. Western blotting using the monoclonal anti-TFPI-2 28Aa antibody (13) revealed that TFPI-2 was expressed in 8 out of the 11 CCC cell lines examined (Fig. 1A). All eight cell lines showed TFPI-2 expression in ECF and four cell lines also expressed TFPI-2 in the WCF. In all cell lines, TFPI-2 was much more abundant in ECF than in WCF. We next fractionated TFPI-2 containing WCFs of the four cell lines into nuclear and cytoplasmic fractions. TFPI-2 was detected in both cytoplasmic (CP) and nuclear fractions (NE) (Fig. 1B). TFPI-2 polypeptides of three molecular weights $(27,31,33 \mathrm{kDa})(12)$ were observed in all 3 fractions, but the larger two molecules were predominant (Fig. 1B). Three cell lines did not express TFPI-2 in any fraction. We also examined TFPI-2 concentration in CM (Fig. 1C). The amount of secreted TFPI-2 in the CM was generally correlated to the levels in ECF. RMG-1 and OVMANA cells strongly expressed TFPI-2 in ECF by western blotting. In contrast, TFPI-2 concentration was high in CM in RMG-1 cells but low in OVMANA cells.

Immunohistochemical analysis of TFPI-2 expression in surgically removed EOC tissues. FFPE samples prepared from 142 patients including 77 OCCC and 65 non-CCC EOC cases were subjected to immunohistochemistry (IHC). The patient clinical information is shown in Table I. The mean age of patients at surgery was 57 years (range 36-84 years).

Experiments with placental tissue confirmed that the antibody stained the cytoplasm of syncytiotrophoblasts, as reported previously (13) (Fig. 2A). We confirmed the specificity of the antibody by an absorption test using the immunized antigen for the 28Aa antibody (Fig. 2B). IHC revealed TFPI-2 in the cytoplasm of tumor cells and in the ECM of OCCC tissues (Fig. 2C and D). We did not detect any nuclear TFPI-2 staining using the 28Aa antibody. Therefore, we next assessed the localization of TFPI-2 using another TFPI-2 antibody (B-7). We confirmed that the B-7 antibody also stained the cytoplasm of syncytiotrophoblasts in placental tissue (Fig. 3A). The specificity of the B-7 antibody was confirmed by absorption test (Fig. 3B). We detected TFPI-2 both in the nucleus and cytoplasm with the B-7 antibody (Fig. 3C); however, signals in ECM were weaker than in staining with the 28Aa antibody (Figs. 2D and 3D). Therefore, we decided to use the B-7 antibody to evaluate nuclear and cytoplasmic expression of TFPI-2, while the 28Aa antibody was used to evaluate TFPI-2 expression in ECM. 

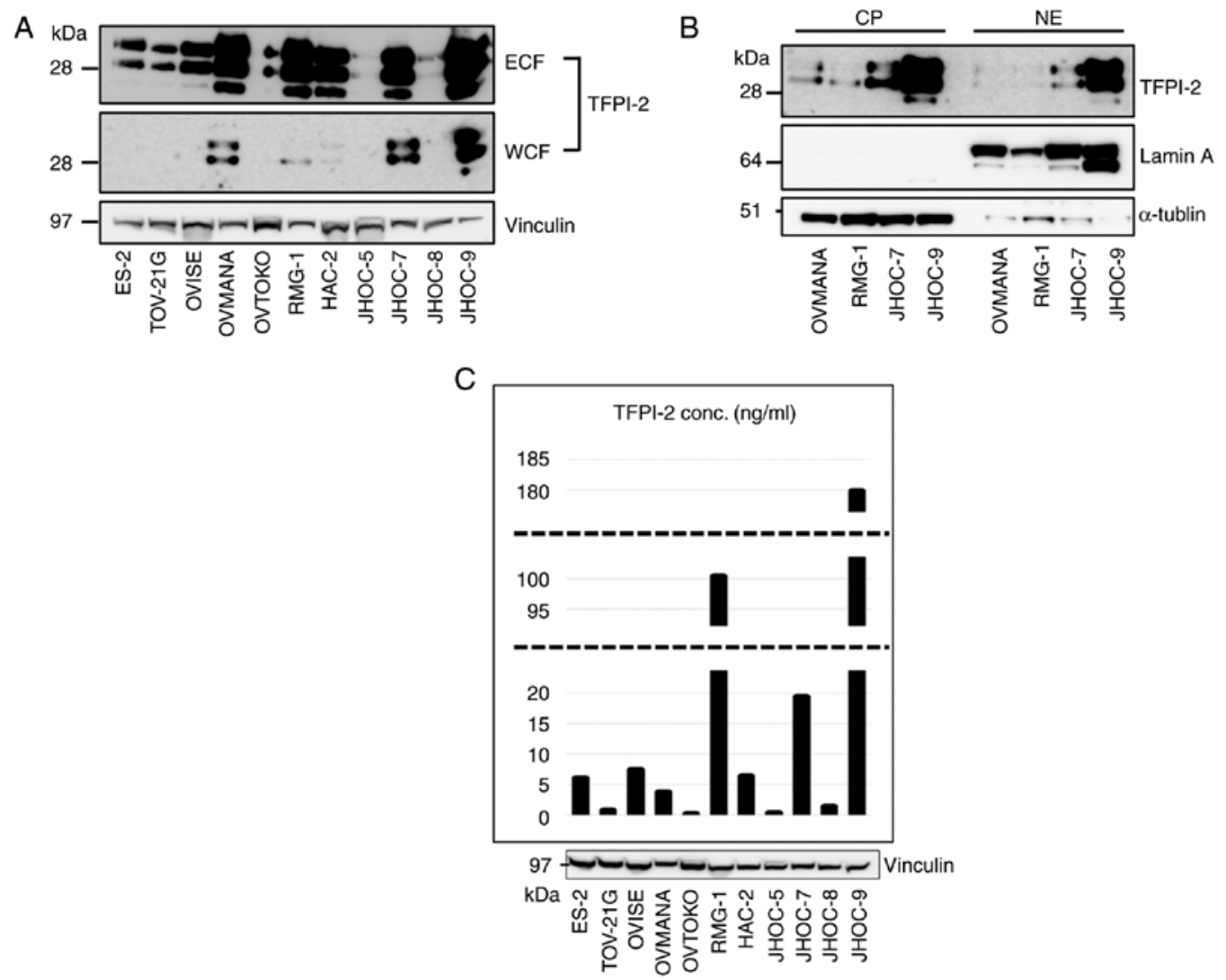

Figure 1. Western blotting and enzyme-linked immune assay of TFPI-2. (A) Western blotting of TFPI-2 expression in 11 cell lines. Vinculin was used for WCF loading control. (B) WCFs were further divided into nuclear and cytoplasm fractions. Lamin A and $\alpha$-tubulin were used for nucleus and cytoplasm markers, respectively. (C) TFPI-2 concentration in CM was assessed by enzyme-linked immune assay. TFPI-2, tissue factor pathway inhibitor-2; ECF, extracellular fraction; WCF, whole cell fraction; NE, nuclear extract; CP, cytoplasm; CM, conditioned medium.

The H-score method using automated scoring software was applied to evaluate TFPI-2 staining (Fig. S1). The H-scores and staining categorization of EOC tissues are shown in Table II. Among OCCC cases, 52/77 (67.5\%) specimens were positive for TFPI-2; among these samples, 35/77 (45.5\%) showed cytoplasmic staining, 10/77 (13.0\%) showed nuclear staining and 35/77 (45.5\%) showed staining in ECM (shown as a Venn diagram in Fig. S2). All cases with positive nuclear staining also showed positive staining in the cytoplasm, and 7/77 (9.1\%) cases showed positive staining in all three fractions (Fig. S2). In contrast, TFPI- 2 was not detected in any of the non-CCC cases (Fig. 4A-C). TFPI-2 expression levels evaluated by IHC distinguished CCC from non-CCC with $67.5 \%$ sensitivity and $100 \%$ specificity. Previous studies showed that TFPI- 2 is expressed in endometrium $(30,31)$. Therefore, we next performed IHC for the non-tumor samples using B-7 antibody in the same manner. Out of 18 cases, 17 cases were negative for TFPI-2 in endometrium cells (Fig. 5A). In one case (5.6\%), endometrium cells were focally positive for TFPI-2. Fallopian tube epithelial cells were all negative for TFPI-2 expression (Fig. 5B).

We next statistically analyzed the correlations between TFPI-2 cytoplasmic expression and clinicopathological characteristics of the OCCC patients according to previous studies $(32,33)$. We examined patient age, parity, menopausal status, rate of elevated serum CA125 level $(>35 \mathrm{U} / \mathrm{ml})$ and distribution of cancer stage (FIGO: International Federation of Gynecology and Obstetrics staging and TNM classification) in univariate analysis according to the cytoplasmic expression status for TFPI-2 (Table III). The median patient age was significantly younger for patients positive for TFPI- 2 than for patients negative for TFPI-2 (56 vs. 60.5 years, respectively; $\mathrm{P}=0.019$ ). Parity, menopausal status, rate of elevated serum level of CA125, FIGO and TNM staging did not significantly correlate with TFPI-2 expression. Kaplan-Meier analysis showed that the 5-year overall survival was not significantly affected by TFPI-2 expression ( $\mathrm{P}=0.621, \log$-rank test) (Fig. S3A). Multivariate analysis revealed that TFPI-2 expression was not an independent prognostic factor (Table SI). Analyses with nuclear and ECM TFPI-2 expression showed similar results (Fig. S3B-D, Tables SI-SIV).

\section{Discussion}

In the present study, we found that tissue factor pathway inhibitor-2 (TFPI-2) is expressed in surgically removed ovarian clear cell carcinoma (OCCC) tissues. We previously identified TFPI-2 as a CCC biomarker using secretome-based analysis of CM derived from OCCC cell lines $(25,26)$ and reported that TFPI-2 may be a useful serum biomarker for OCCC patients. The confirmation of TFPI-2 expression in OCCC tumor cells in surgical tissues using IHC strongly supports the development of TFPI-2 as a serum tumor biomarker.

We demonstrated that TFPI-2 is localized in the nucleus as well as the cytoplasm and extracellular fraction (ECF) 
Table I. Clinicopathological characteristics of the 142 epithelial ovarian cancer patients.

\begin{tabular}{|c|c|c|c|c|c|c|}
\hline Characteristics & OCCC $(n=77)$ & $\mathrm{SC}(\mathrm{n}=20)$ & EMC (n=19) & MOC (n=17) & Others $(n=9)$ & P-value \\
\hline Period (year) & 2005 to 2017 & 2014 to 2017 & 2011 to 2017 & 2005 to 2017 & 2014 to 2017 & \\
\hline Age in years, median (range) & $58(36-75)$ & $67.5(37-80)$ & $54(38-83)$ & $55(38-84)$ & $60(47-83)$ & $\mathrm{P}=0.0875$ \\
\hline Parity (\%) & & & & & & $\mathrm{P}=0.024$ \\
\hline No $(0)$ & $36(46.8)$ & $4(20.0)$ & $7(36.8)$ & $3(17.6)$ & $6(66.7)$ & \\
\hline Yes $(\geq 1)$ & $41(53.2)$ & $16(80.0)$ & $12(63.1)$ & $14(82.4)$ & $3(33.3)$ & \\
\hline Menopausal status (\%) & & & & & & $\mathrm{P}=0.149$ \\
\hline Premenopause & $18(23.4)$ & $2(10.0)$ & $7(36.8)$ & $7(41.2)$ & $3(33.3)$ & \\
\hline Postmenopause & $59(76.6)$ & $18(90.0)$ & $12(63.1)$ & $10(58.8)$ & $6(66.7)$ & \\
\hline CA125 (\%) & & & & & & $\mathrm{P}=0.321$ \\
\hline$<35$ & $24(31.2)$ & $2(10.0)$ & $7(36.8)$ & $5(29.4)$ & $2(22.2)$ & \\
\hline$\geq 35$ & $53(68.8)$ & $18(90.0)$ & $12(63.1)$ & $12(70.6)$ & $7(77.8)$ & \\
\hline FIGO (\%) & & & & & & $\mathrm{P}<0.001$ \\
\hline $\mathrm{I} / \mathrm{II}$ & $61(79.2)$ & $3(15.0)$ & $17(89.4)$ & $16(94.1)$ & $5(55.6)$ & \\
\hline III/IV & $16(20.8)$ & $17(85.0)$ & $2(10.5)$ & $1(5.9)$ & $4(44.4)$ & \\
\hline Site of specimen $(\%)$ & & & & & & $\mathrm{P}<0.001$ \\
\hline Primary site & $77(100)$ & $17(85.0)$ & $19(100)$ & $17(100)$ & $7(77.8)$ & \\
\hline Omentum & $0(0)$ & $3(15.0)$ & $0(0)$ & $0(0)$ & $2(22.2)$ & \\
\hline
\end{tabular}

OCCC, ovarian clear cell carcinoma; SC, serous carcinoma; EMC, endometrioid carcinoma; MOC, mucinous ovarian carcinoma. Others: Two carcinosarcoma, one squamous cell carcinoma arising from mature cystic teratoma, one small cell carcinoma, one adenofibrocarcinoma, one mixed epithelial tumor, one large cell neuroendocrine carcinoma, one undifferentiated and one adenocarcinoma (not otherwise specified). Kruskal-Wallis test was used for age. Fisher's exact test was used for other parameters. CA125, cancer antigen 125; FIGO, Federation of Gynecology and Obstetrics.
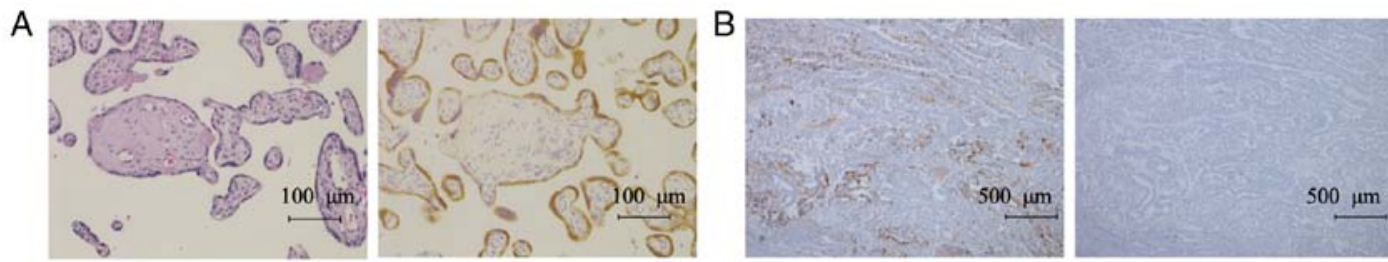

C
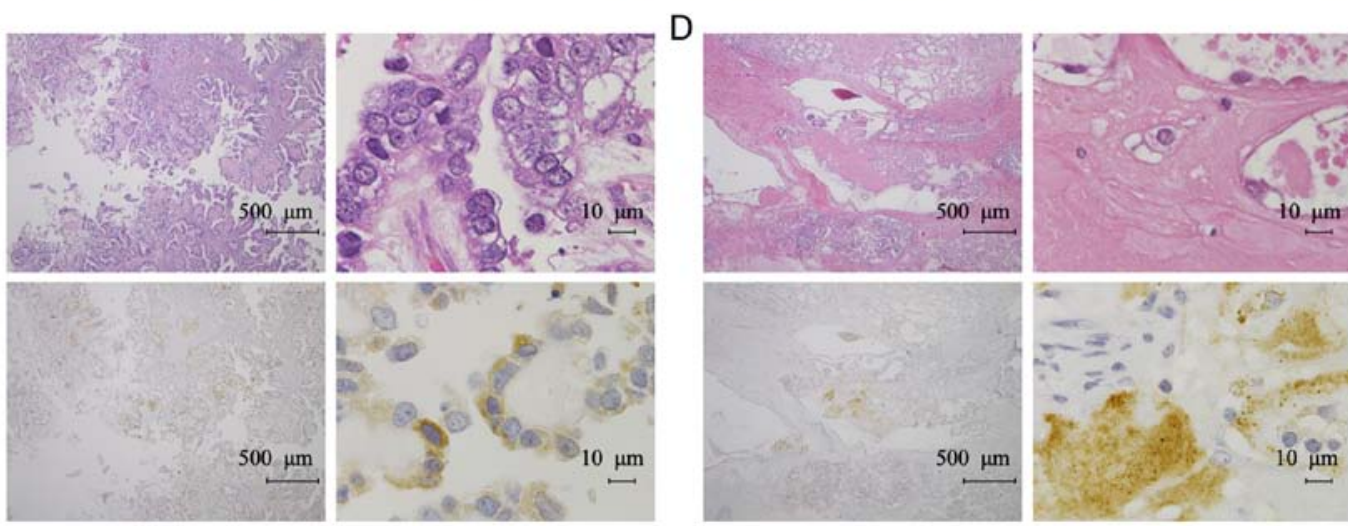

Figure 2. IHC analysis of TFPI-2 subcellular localization. (A) Typical H\&E staining (left) and TFPI-2 staining using the 28 Aa antibody (right) in placenta specimens. (B) Specificity of TFPI-2 antibody was confirmed in an OCCC specimen by absorption test using the 14 amino acid peptide antigen corresponding to the N-terminus of mature TFPI-2 protein. Left: No antigen control. Right: Experiment with pre-absorbed antibody. (C) Typical H\&E staining (top) and cytoplasmic TFPI-2 staining (bottom) patterns in an OCCC specimen. (D) Typical H\&E staining (top) and TFPI-2 staining (bottom) patterns in a stromal lesion of an OCCC specimen. IHC, immunohistochemistry; TFPI-2, tissue factor pathway inhibitor-2; H\&E, hematoxylin and eosin; OCCC, ovarian clear cell carcinoma.

of cultivated OCCC cells. TFPI-2 has been characterized as a secreted protein (23) that contains a signal peptide at its N-terminus, and mature TFPI-2 protein is secreted into the ECF through the endoplasmic reticulum and secretory pathway $(11,34)$. A recent study, however, showed that TFPI- 2 was also localized in the nucleus and cytoplasm in 
Table II. TFPI-2 expression score according to subcellular localization.

\begin{tabular}{lccc}
\hline Subcellular localization & H-score & CCC $(\mathrm{n}=77) \mathrm{n}(\%)$ & Non-CCC $(\mathrm{n}=65) \mathrm{n}(\%)$ \\
\hline Nuclear & 0 & $67(87.0)$ & $65(100)$ \\
Negative & $1-9$ & $4(1.3)$ & $0(0)$ \\
Positive & $10-29$ & $5(5.2)$ & $0(0)$ \\
& $30-$ & $5(6.5)$ & $0(0)$ \\
Cytoplasm & & $20(26.0)$ & $65(100)$ \\
Negative & 0 & $9(11.7)$ & $0(0)$ \\
Positive & $1-9$ & $6(7.8)$ & $0(0)$ \\
& $10-29$ & & $0(0)$ \\
ECM & $30-$ & $42(54.5)$ & $65(100)$ \\
Negative & & $35(45.5)$ & $0(0)$ \\
Positive & & & \\
\hline
\end{tabular}

Cut-off for positive/negative expression is H-score=1. TFPI-2, tissue factor pathway inhibitor-2; CCC, clear cell carcinoma; ECM, extracellular matrix.

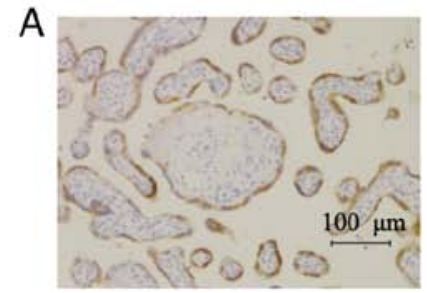

C

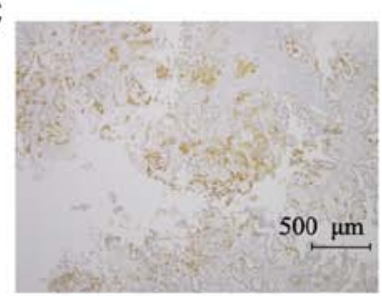

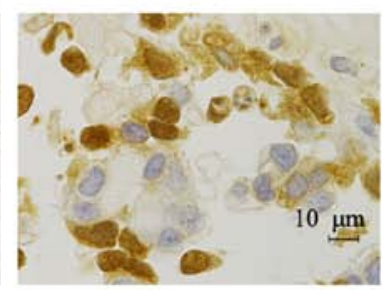
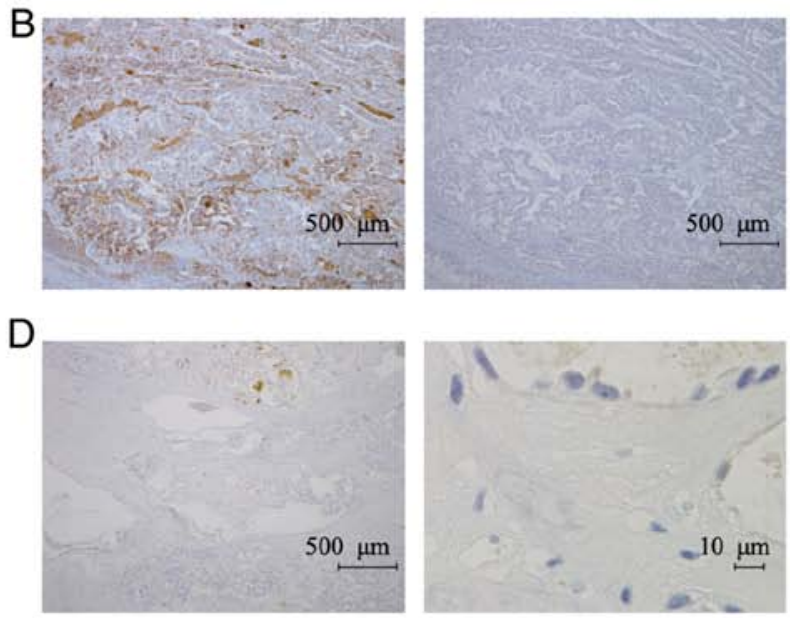

Figure 3. IHC analysis of TFPI-2 using the B-7 antibody. (A) Typical TFPI-2 staining in a placenta specimen using the B-7 antibody. TFPI-2 was detected in the syncyiotrophoblasts. (B) B-7 antibody specificity was confirmed in an OCCC specimen by absorption test using recombinant full-length TFPI-2 protein as an antigen. Left: No antigen control. Right: Experiment with pre-absorbed antibody. (C) B-7 antibody stains the nucleus and cytoplasm in the same sample shown in Fig. 2C. (D) B-7 antibody failed to stain ECM in the same sample shown in Fig. 2D. IHC, immunohistochemistry; TFPI-2, tissue factor pathway inhibitor-2; OCCC, ovarian clear cell carcinoma; ECM, extracellular matrix.

endothelial cell lines (23), and TFPI-2 exogenously added to culture medium in vitro was rapidly internalized and distributed in both nucleus and cytoplasmic fractions. A nuclear localization signal was found in the C-terminal tail of TFPI-2 (23). In the nucleus, TFPI-2 regulates MMP-2 gene transcription through the interaction with AP-2a, a transcription factor important for the expression of many genes (24). In the cytoplasm, TFPI-2 regulates ERK signaling and interacts with a-actinin-4 and myosin-9, resulting in increased cancer cell activities (35). Consistent with the in vitro study, we confirmed the nuclear, cytoplasm, and extracellular matrix (ECM) subcellular localization of TFPI-2 in surgically resected OCCC tissues. We detected TFPI-2 mainly in the ECF in vitro; however, the four cell lines with the highest expression of TFPI-2 also expressed TFPI-2 in both the nucleus and cytoplasm. Three different molecular sized TFPI-2 polypeptides, which are speculated to be derived from differential glycosylation events (12), were detected in all three fractions. Taken together, these findings suggest that mature TFPI-2, after cleavage of the signal peptide and posttranslational modifications, might be retained in the cytoplasm or internalized after secretion and distributed into the cytoplasm or nucleus when large amounts of TFPI-2 are produced. In OCCC OVMANA cells, the level of secreted TFPI-2 was not as high as its expression in ECF. In contrast, the majority of secreted TFPI-2 in ES-2 cells seemed to be retained in the medium. The mechanisms regulating TFPI-2 localization remain to be elucidated. 
Table III. Clinicopathological characteristic and TFPI-2 cytoplasmic expression in 77 CCC samples.

\begin{tabular}{|c|c|c|c|}
\hline Characteristics & Negative $(n=42)$ & Positive $(n=35)$ & P-value \\
\hline Age in years, median (range) & $60.5(36-74)$ & $56(39-75)$ & $\mathrm{P}=0.019$ \\
\hline \multicolumn{4}{|l|}{ Parity, n (\%) } \\
\hline No $(0)$ & $20(47.6)$ & $16(45.7)$ & \\
\hline Yes $(\geq 1)$ & $22(52.4)$ & $19(54.3)$ & $\mathrm{P}=0.990$ \\
\hline \multicolumn{4}{|l|}{ Menopausal status, n (\%) } \\
\hline Premenopause & $7(16.7)$ & $11(31.4)$ & \\
\hline Postmenopause & $35(83.3)$ & $24(68.6)$ & $\mathrm{P}=0.177$ \\
\hline \multicolumn{4}{|l|}{ CA125 (U/ml), n (\%) } \\
\hline$<35$ & $13(31.0)$ & $11(31.4)$ & \\
\hline$\geq 35$ & $29(69.0)$ & $24(68.6)$ & $\mathrm{P}=0.990$ \\
\hline \multicolumn{4}{|l|}{ FIGO,n (\%) } \\
\hline $\mathrm{I} / \mathrm{II}$ & $32(76.2)$ & $29(82.9)$ & \\
\hline III/IV & $10(23.8)$ & $6(17.1)$ & $\mathrm{P}=0.577$ \\
\hline \multicolumn{4}{|l|}{ pT } \\
\hline $\mathrm{pT} 1 / 2$ & $33(78.6)$ & $29(82.9)$ & \\
\hline pT3 & $9(21.4)$ & $6(17.1)$ & $\mathrm{P}=0.775$ \\
\hline \multicolumn{4}{|l|}{$\mathrm{pN}$} \\
\hline pNO & $8(19.0)$ & $4(11.4)$ & \\
\hline $\mathrm{pN} 1$ & $1(2.4)$ & $0(0)$ & \\
\hline $\mathrm{pNx}$ & $33(78.6)$ & $31(88.6)$ & $\mathrm{P}=0.441$ \\
\hline \multicolumn{4}{|l|}{ M } \\
\hline M0 & $41(97.6)$ & $33(94.3)$ & \\
\hline M1 & $1(2.4)$ & $2(5.7)$ & $\mathrm{P}=0.588$ \\
\hline
\end{tabular}

Mann-Whitney U test was used for continuous variables. Fisher's exact test was used for non-continuous variables. TFPI-2, tissue factor pathway inhibitor-2; CCC, clear cell carcinoma; CA125, cancer antigen 125; FIGO, Federation of Gynecology and Obstetrics.

In this study, we demonstrated the specificity of TFPI-2 for $\mathrm{CCC}$ in IHC. CCC is pathologically diagnosed based on morphologic features such as hobnail cells with clear cytoplasm (3). However, tumors containing clear cells with heterogeneous features are not reproducibly diagnosed (3). Currently, hepatocyte nuclear factor-1 $\beta$ (HNF-1 $\beta$ ) immunohistochemical expression (sensitivity, 82.5-85.2\%; specificity, 76.5-95.2\%) (36,37), Napsin A (38) and glypican-3 (39) are candidates for CCC IHC markers. In this study, we showed that TFPI-2 was only identified in CCC tissues and not in non-CCC EOC tissues. This result is well consistent with The Human Protein Atlas data, which examined TFPI-2 expression in limited numbers of EOC surgical specimens by IHC but did not detect any cases with positive TFPI- 2 expression (serous $0 / 5$, mucinous $0 / 4$, endometrioid 0/2 cases; CCC cases were not enrolled) (40). Our results showed that TFPI-2 expression distinguished CCC from non-CCC with a sensitivity of $67.5 \%$ and specificity of $100 \%$. The high specificity of TFPI-2 may support its use for diagnosis of OCCC in combination with existing markers. We propose TFPI-2 as an IHC biomarker for histopathological diagnostics as well as serum biomarker for OCCC patients.

We found that all serous carcinoma cases in the current study group were negative for TFPI-2 in IHC. We previously showed that serum TFPI-2 levels greater than $345 \mathrm{pg} / \mathrm{ml}$ can pre-operatively discriminate OCCC from other EOC subtypes and borderline ovarian tumors with a sensitivity of $71.4 \%$ and specificity of $85.7 \%(25,26)$. Additionally, we found that serum TFPI-2 level was also increased in $29.4 \%$ of serous carcinoma patients (26). In this study, all serous carcinoma cases were negative for TFPI-2 despite setting the H-score cut-off value very low. Considering our IHC results, we speculate that the elevation of TFPI- 2 in the serum of serous carcinoma patients was derived from non-tumor cells such as endothelial cells (23) or platelets (41), although the numbers of examined serous carcinoma cases were limited and the putative mechanisms are currently unclear.

We then examined the clinical significance of TFPI-2 expression in OCCC tissues but did not identify any significant association between TFPI-2 expression in the primary site and aggressiveness of the OCCC cases. This is not consistent with published data from other cancer types, which showed that low expression of TFPI-2 in IHC is associated with poor survival in breast and pancreatic cancer patients $(32,33)$. The tumor suppressor-like activity of TFPI-2 suggested by these reports are consistent with in vitro and animal experiments showing that secreted TFPI- 2 reduces invasiveness, through preventing ECM degeneration by inhibiting proteases, such as plasmin or MMPs $(42,43)$. In many cancer types, TFPI- 2 expression is 

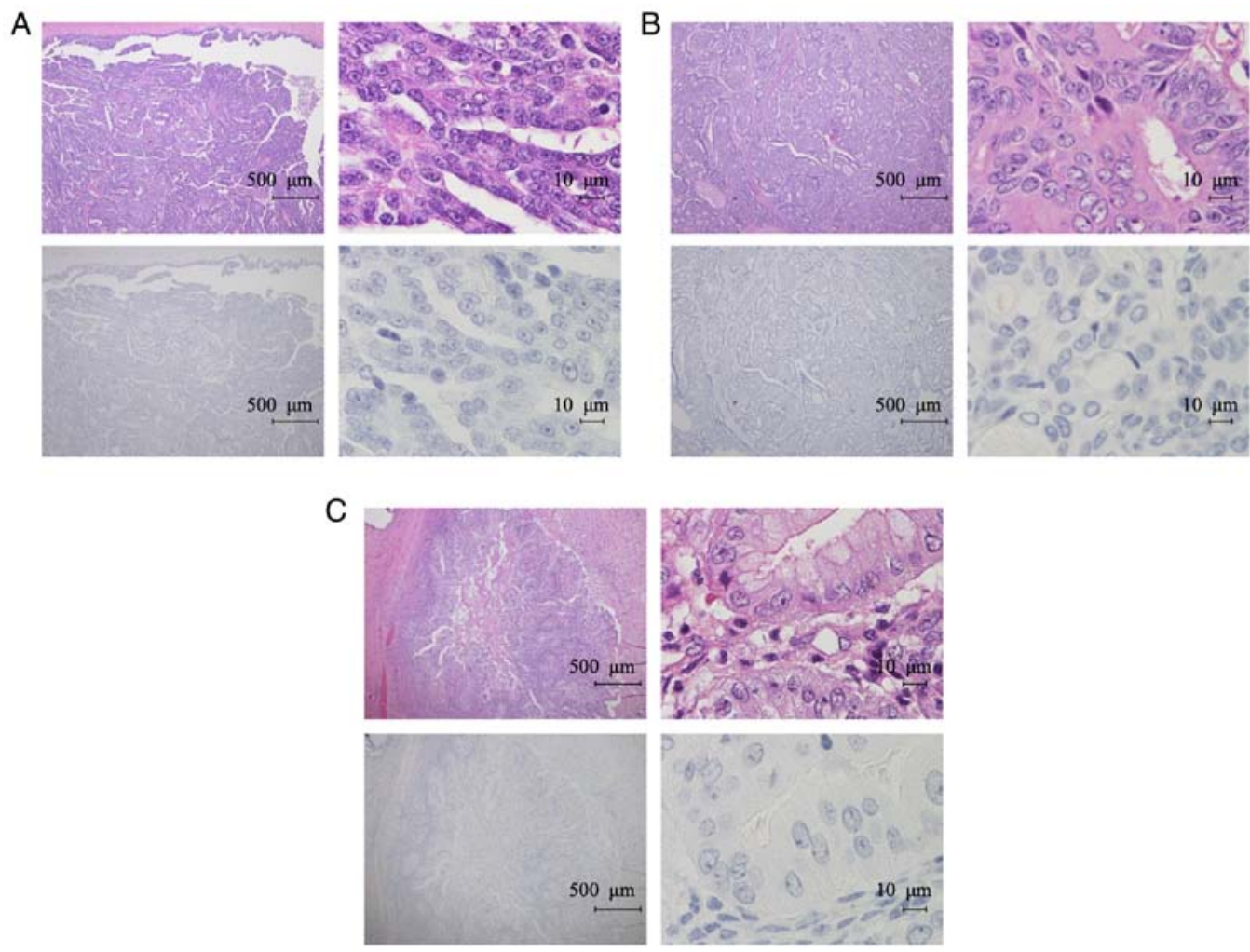

Figure 4. IHC analysis of TFPI-2 in non-CCC EOC tissues. Representative images of H\&E staining (top) and IHC for TFPI-2 (bottom) in non-CCC tissues. (A) High grade serous carcinoma. (B) Endometrioid carcinoma. (C) Mucinous carcinoma. IHC, immunohistochemistry; TFPI-2, tissue factor pathway inhibitor-2; CCC, clear cell carcinoma; EOC, epithelial ovarian cancer; H\&E, hematoxylin and eosin.
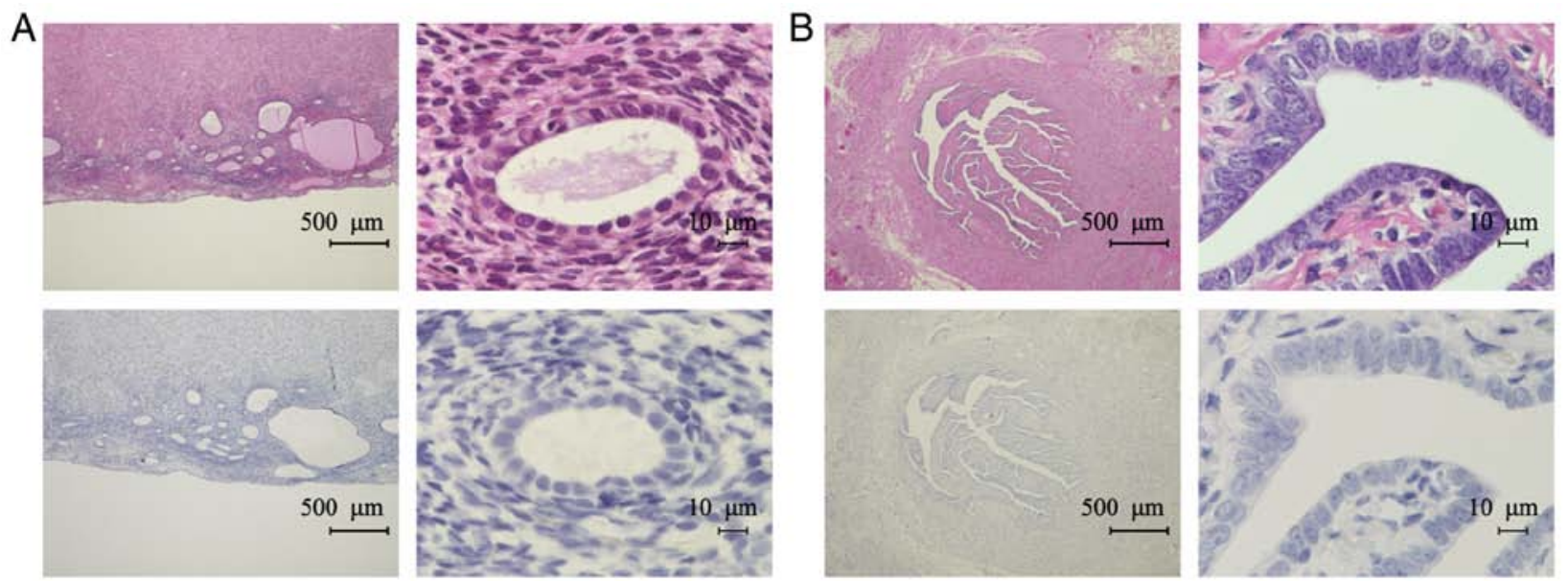

Figure 5. IHC analysis of TFPI-2 in non-neoplastic tissues. Representative images of H\&E staining (top) and IHC for TFPI-2 (bottom) in non-neoplastic tissues. (A) Endometrium of TFPI-2-negative case. (B) Fallopian tube epithelium. IHC, immunohistochemistry; TFPI-2, tissue factor pathway inhibitor-2; H\&E, hematoxylin and eosin.

epigenetically silenced by aberrant methylation of $\mathrm{CpG}$ islands in the TFPI-2 promoter $(16,20)$. In contrast, our study showed that TFPI-2 is elevated in the serum of OCCC patients and is certainly expressed in OCCC tumor cells. These findings suggest that the roles of TFPI-2 may vary depending on the cancer type and that the function of TFPI- 2 in ovarian CCC is unique compared with its role in other cancers. In this study, we excluded cases that received neoadjuvant therapies to precisely evaluate the TFPI- 2 expression dynamics in OCCC tissues, and therefore the enrolled patients were predicted to have an inherent good prognosis and likely to be in early stages. This bias could be another possibility to explain the negative correlation of TFPI-2 expression and clinical aggressiveness in OCCC tissue. Further studies are needed to elucidate the potential value of TFPI-2 as a prognostic marker or monitoring marker for OCCC patients.

In conclusion, we confirmed the expression of TFPI- 2 in clinical OCCC tissues and confirmed the nuclear, cytoplasm, and ECF/ECM subcellular localization of TFPI-2 in cultivated OCCC cells and surgical tissues. We also demonstrated the 
high specificity of TFPI-2 expression in OCCC tissues. TFPI-2 expression in IHC may support its use for diagnosis of OCCC in combination with existing markers.

\section{Acknowledgements}

The authors would like to express our appreciation to Masahiko Sakaguchi for his valuable and constructive suggestions for statistical analysis. We would also like to thank the members of the Department of Gynecology and the Department of Pathology of Kanagawa Cancer Center Hospital for their cooperation with this research.

\section{Funding}

This study was funded by Tosoh Corporation, Japan. SM and NO are employees of the Tosoh Corporation. SM and NO provided technical support for the experiments by analyzing TFPI-2 concentration in CM. The submission fee was provided by Tosoh Corporation. EM obtained a grant from Tosoh Corporation, outside the submitted work. YM obtained grants from Tosoh Corporation, both for this work and outside the submitted work.

\section{Availability of data and materials}

The datasets generated and analyzed during the current study are available from the corresponding author on reasonable request. Aperio's annotation software is available at https://www.leicabiosystems.com/digital-pathology/Accessed 13/07/2010.

\section{Authors' contributions}

YO contributed to the methodology, software, formal analysis, investigation, and writing of the original draft. SK contributed to the methodology, writing of the review and editing. YN contributed to the investigation. MY contributed to the investigation. TT contributed to the investigation. SS contributed to the investigation. SM contributed to the investigation. NO contributed to the investigation. HK contributed the resources and conducted the data curation. TY conducted the validation and contributed to the resources. EM was responsible for the conceptualization and supervision. YM contributed to the conceptualization, validation and writing of the review and editing as well as the supervision. All authors read and approved the manuscript and agree to be accountable for all aspects of the research in ensuring that the accuracy or integrity of any part of the work are appropriately investigated and resolved.

\section{Ethics approval and consent to participate}

The experimental protocol of the present study was reviewed and approved by the Institutional Review Board of Kanagawa Cancer Center Hospital (approval no. ethics-2018-10). Written informed consent was obtained from the patients for publication of the study and accompanying images.

\section{Patient consent for publication}

Not applicable.

\section{Competing interests}

SM and NO are employees of the Tosoh Corporation, which is now developing an in vitro diagnosis approach for ovarian CCC patients by evaluating blood TFPI-2 concentration. EM obtained a grant from Tosoh Corporation, outside the submitted work. YM obtained grants from Tosoh Corporation, both for this work and outside the submitted work. The other authors have no conflicts of interest directly relevant to the content of this article.

\section{Authors' information}

ORCID: Yukihide Ota: 0000-0002-5167-1918; Shiro Koizume: 0000-0002-9132-5286; Etsuko Miyagi: 0000-0002-5492-0844.

\section{References}

1. Siegel RL, Miller KD and Jemal A: Cancer statistics. CA Cancer J Clin 68: 7-30, 2018.

2. World Health Organization, International Agency for Research on Cancer, Cancer Fact Sheets. http://gco.iarc. fr/today/data/factsheets/cancers/25-Ovary-fact-sheet.pdf, 2018 Accessed 4, September 2020.

3. Soslow RA: Histologic subtypes of ovarian carcinoma: An overview. Int J Gynecol Pathol 27: 161-174, 2008.

4. Machida H, Matsuo K, Yamagami W, Ebina Y, Kobayashi Y, Tabata T, Kanauchi M, Nagase S, Enomoto T and Mikami M: Trends and characteristics of epithelial ovarian cancer in Japan between 2002 and 2015: A JSGO-JSOG joint study. Gynecol Oncol 153: 589-596, 2019.

5. Lee AW, Navajas EE and Liu L: Clear differences in ovarian cancer incidence and trends by ethnicity among Asian Americans. Cancer Epidemiol 61: 142-149, 2019.

6. Shu CA, Zhou Q, Jotwani AR, Iasonos A, Leitao MM Jr, Konner JA and Aghajanian CA: Ovarian clear cell carcinoma, outcomes by stage: The MSK experience. Gynecol Oncol 139: 236-241, 2015.

7. Anglesio MS, Carey MS, Köbel M, Mackay H and Huntsman DG; Vancouver Ovarian Clear Cell Symposium Speakers: Clear cell carcinoma of the ovary: A report from the first ovarian clear cell symposium, June 24th, 2010. Gynecol Oncol 121: 407-415, 2011.

8. Meyer T and Rustin GJ: Role of tumour markers in monitoring epithelial ovarian cancer. Br J Cancer 82: 1535-1538, 2000.

9. Kudoh K, Kikuchi Y, Kita T, Tode T, Takano M, Hirata J, Mano Y, Yamamoto K and Nagata I: Preoperative determination of several serum tumor markers in patients with primary epithelial ovarian carcinoma. Gynecol Obstet Invest 47: 52-57, 1999.

10. Sierko E, Wojtukiewicz MZ and Kisiel W: The role of tissue factor pathway inhibitor-2 in cancer biology. Semin Thromb Hemost 33: 653-659, 2007.

11. Miyagi Y, Koshikawa N, Yasumitsu H, Miyagi E, Hirahara F, Aoki I, Misugi K, Umeda M and Miyazaki K: cDNA cloning and mRNA expression of a serine proteinase inhibitor secreted by cancer cells: Identification as placental protein 5 and tissue factor pathway inhibitor-2. J Biochem 116: 939-942, 1994.

12. Rao CN, Reddy P, Liu Y, O'Toole E, Reeder D, Foster DC, Kisiel W and Woodley DT: Extracellular matrix-associated serine protease inhibitors $(\mathrm{Mr} 33,000,31,000$, and 27,000) are single-gene products with differential glycosylation: cDNA cloning of the 33-kDa inhibitor reveals its identity to tissue factor pathway inhibitor-2. Arch Biochem Biophys 335: 82-92, 1996.

13. Ogawa M, Yanoma S, Nagashima Y, Okamoto N, Ishikawa H, Haruki A, Miyagi E, Takahashi T, Hirahara F and Miyagi Y: Paradoxical discrepancy between the serum level and the placental intensity of PP5/TFPI-2 in preeclampsia and/or intrauterine growth restriction: Possible interaction and correlation with glypican-3 hold the key. Placenta 28: 224-232, 2007.

14. Karaszi K, Szabo S, Juhasz K, Kiraly P, Kocsis-Deak B, Hargitai B, Krenacs T, Hupuczi P, Erez O, Papp Z, et al: Increased placental expression of placental protein 5 (PP5)/tissue factor pathway inhibitor-2 (TFPI-2) in women with preeclampsia and HELLP syndrome: Relevance to impaired trophoblast invasion? Placenta 76: 30-39, 2019. 
15. Rao CN, Lakka SS, Kin Y, Konduri SD, Fuller GN, Mohanam S and Rao JS: Expression of tissue factor pathway inhibitor 2 inversely correlates during the progression of human gliomas. Clin Cancer Res 7: 570-576, 2001

16. Rollin J, Iochmann S, Bléchet C, Hubé F, Régina S, Guyétant S, Lemarié E, Reverdiau P and Gruelet Y: Expression and methylation status of tissue factor pathway inhibitor- 2 gene in non-small-cell lung cancer. Br J Cancer 92: 775-783, 2005.

17. Sato N, Parker AR, Fukushima N, Miyagi Y, Iacobuzio-Donahue CA, Eshleman JR and Gogginset $M$ : Epigenetic inactivation of TFPI-2 as a common mechanism associated with growth and invasion of pancreatic ductal adenocarcinoma. Oncogene 24: 850-858, 2005

18. Guo H, Lin Y, Zhang H, Liu J, Zhang N, Li Y, Kong D, Tang Q and Ma D: Tissue factor pathway inhibitor-2 was repressed by CpG hypermethylation through inhibition of KLF6 binding in highly invasive breast cancer cells. BMC Mol Biol 8: 110, 2007.

19. Nobeyama Y, Okochi-takada E, Furuta J, Miyagi Y, Kikuchi K, Yamamoto A, Nakanishi Y, Nakagawa $\mathrm{H}$ and Ushijima $\mathrm{T}$ : Silencing of tissue factor pathway inhibitor-2 gene in malignant melanomas. Int J Cancer 121: 301-307, 2007.

20. Wong C, Ng Y, Lee JM, Wong CC, Cheung O, Chan C, Tung EK, Ching $\mathrm{Y}$ and $\mathrm{Ng}$ IO: Tissue factor pathway inhibitor-2 as a frequently silenced tumor suppressor gene in hepatocellular carcinoma. Hepatology 45: 1129-1138, 2007.

21. Xu Y, Qin X, Zhou J, Tu Z, Bi X, Li W, Fan X and Zhang Y: Tissue factor pathway inhibitor-2 inhibits the growth and invasion of hepatocellular carcinoma cells and is inactivated in human hepatocellular carcinoma. Oncol Lett 2: 779-783, 2011.

22. Lavergne M, Jourdan ML, Blechet C, Guyetant S, Pape AL, Heuze-Vourc'h N, Courty Y, Lerondel S, Sobilo J, Iochmann S and Reverdiau P: Beneficial role of overexpression of TFPI-2 on tumour progression in human small cell lung cancer. FEBS Open Bio 3: 291-301, 2013.

23. Kempaiah $\mathrm{P}$, Chand $\mathrm{HS}$ and Kisiel W: Human tissue factor pathway inhibitor- 2 is internalized by cells and translocated to the nucleus by the importin system. Arch Biochem Biophys 482: 58-65, 2009.

24. Wang G, Zeng Y, Chen S, Li D, Li W, Zhou Y, Singer RH and $\mathrm{Gu}$ W: Localization of TFPI-2 in the nucleus modulates MMP-2 gene expression in breast cancer cells. Sci Rep 7: 13575, 2017.

25. Arakawa N, Miyagi E, Nomura A, Morita E, Ino Y, Ohtake N, Miyagi Y, Hirahara F and Hirano H: Secretome-Based identification of TFPI2, A novel serum biomarker for detection of ovarian clear cell adenocarcinoma. J Proteome Res 12: 4340-4350, 2013

26. Arakawa N, Kobayashi H, Yonemoto N, Masuishi Y, Ino Y, Shigetomi H, Furukawa N, Ohtake N, Miyagi Y, Hirahara F, et al: Clinical significance of tissue factor pathway inhibitor 2, a serum biomarker candidate for ovarian clear cell carcinoma. PLoS One 11: e0165609, 2016.

27. Pearce CL, Templeman C, Rossing MA, Lee A, Near AM, Webb PM, Nagle CM, Doherty JA, Cushing-Haugen KL, Wicklund KG, et al: Association between endometriosis and risk of histological subtypes of ovarian cancer: A pooled analysis of case-control studies. Lancet Oncol 13: 385-394, 2012.

28. Cinti S, Matteis RD, Picó C, Ceresi E, Obrador A, Maffeis C, Oliver J and Palou A: Secretory granules of endocrine and chief cells of human stomach mucosa contain leptin. Int J Obes Relat Metab Disord 24: 789-793, 2000.

29. Pirker R, Pereira JR, von Pawel J, Krzakowski M, Ramlau R, Park K, de Marinis F, Eberhardt WE, Paz-Ares L, Störkel S, et al: EGFR expression as a predictor of survival for first-line chemotherapy plus cetuximab in patients with advanced non-small-cell lung cancer: Analysis of data from the phase 3 FLEX study. Lancet Oncol 13: 33-42, 2012.
30. Wojtukiewicz MZ, Sierko E, Zimnoch L, Kozlowski L and Kisiel W: Immunohistochemical localization of tissue factor pathway inhibitor-2 in human tumor tissue. Thromb Haemost 90: 140-146, 2003.

31. Altmäe S, Salumets A, Bjuresten K, Kallak TK, Wånggren K, Landgren BM, Hovatta $\mathrm{O}$ and Stavreus-Evers A: Tissue factor and tissue factor pathway inhibitors TFPI and TFPI2 in human secretory endometrium-possible link to female infertility. Reprod Sci 18: 666-678, 2011

32. Zhai LL, Cai CY, Wu Y and Tang ZG: Correlation and prognostic significance of MMP-2 and TFPI-2 differential expression in pancreatic carcinoma. Int J Clin Exp Pathol 8: 682-691, 2015

33. Xu C, Wang H, He H, Zheng F, Chen Y, Zhang J, Lin X, Ma D and Zhang H: Low expression of TFPI-2 associated with poor survival outcome in patients with breast cancer. BMC Cancer 13: 118, 2013.

34. Sprecher CA, Kisiel W, Mathewes S and Foster DC: Molecular cloning, expression, and partial characterization of a second human tissue-factor-pathway inhibitor. Proc Natl Acad Sci USA 91: 3353-3357, 1994.

35. Wang G, Huang W, Li W, Chen S, Chen W, Zhou Y, Peng P and Gu W: TFPI-2 suppresses breast cancer cell proliferation and invasion through regulation of ERK signaling and interaction with actinin-4 and myosin-9. Sci Rep 8: 14402, 2018.

36. Köbel M, Kalloger SE, Carrick J, Huntsman D, Asad H, Oliva E, Ewanowich CA, Soslow RA and Gilks CB: A limited panel of immunomarkers can reliably distinguish between clear cell and high-grade serous carcinoma of the ovary. Am J Surg Pathol 33: 14-21, 2009.

37. Huang W, Cheng X, Ji J, Zhang J and Li Q: The application value of HNF-1 $\beta$ transcription factor in the diagnosis of ovarian clear cell carcinoma. Int J Gynecol Pathol 35: 66-71, 2016.

38. Yamashita Y, Nagasaka T, Naiki-Ito A, Sato S, Suzuki S, Toyokuni S, Ito $M$ and Takahashi S: Napsin A is a specific marker for ovarian clear cell adenocarcinoma. Mod Pathol 28: 111-117, 2015.

39. Maeda D, Ota S, Takazawa Y, Aburatani H, Nakagawa S, Yano T, Taketani Y, Kodama T and Fukayama M: Glypican-3 expression in clear cell adenocarcinoma of the ovary. Mod Pathol 22: 824-832, 2009.

40. The Human Protein Atlas: TFPI2. https://www.proteinatlas.org/ ENSG00000105825-TFPI2. Accessed April 8, 2020.

41. Vadivel K, Ponnuraj SM, Kumar Y, Zaiss AK, Bunce MW, Camire RM, Wu L, Evseenko D, Herschman HR, Bajaj MS and Bajaj SP: Platelets contain tissue factor pathway inhibitor-2 derived from megakaryocytes and inhibits fibrinolysis. J Biol Chem 289: 31647-31661, 2014.

42. Rao CN, Cook B, Liu Y, Chilukuri K, Stack MS, Foster DC, Kisiel W and Woodley DT: HT-1080 fibrosarcoma cell matrix degradation and invasion are inhibited by the matrix-associated serine protease inhibitor TFPI-2/33 kDa MSPI. Int J Cancer 76: 749-756, 1998

43. Jin M, Udagawa K, Miyagi E, Nakazawa T, Hirahara F, Yasumitsu H, Miyazaki K, Nagashima Y, Aoki I and Miyagi Y: Expression of serine proteinase inhibitor PP5/TFPI-2/MSPI decreases the invasive potential of human choriocarcinoma cells in vitro and in vivo. Gynecol Oncol 83: 325-333, 2001.

This work is licensed under a Creative Commons Attribution-NonCommercial-NoDerivatives 4.0 International (CC BY-NC-ND 4.0) License. 